\title{
Simulation of Lung Behaviour with Finite Elements : Influence of Bio-Mechanical Parameters
}

\author{
Pierre-Frédéric Villard, Michaël Beuve, Behzad Shariat, Vincent Baudet and Fabrice Jaillet \\ LIRIS, bat Nautibus, 8 bd Niels Bohr, 69622 Villeurbanne CEDEX, FRANCE \\ pierre-frederic.villard@liris.univ-lyon1.fr
}

\begin{abstract}
Motivated by medical needs, we propose to simulate lung deformation and motion during respiration to track tumours. This paper presents a model of lung behaviour based on a continuous media mechanics model and solved with a finite element method. The result is a simulation of a normal breathing, matching with patient customised data. Moreover, we carried out numerical experiments to evaluate our algorithms and to measure the influence and the relevance of mechanical parameters.
\end{abstract}

\section{Introduction}

Radiotherapy and Hadrontherapy are techniques which are efficient to cure local cancerous tumours. They consist in delivering a lethal dose of ionising beam to the cancerous volume, while reducing impact on surrounding healthy tissues. Such treatments need accurate targeting necessitating a precise knowledge of the shape and position of tumours. Concerning lung cancer, breathing motion is then a crucial problem for variety of tumours. Lung displacements need to be estimated.

As mentioned in [3], various approaches have been explored to predict tumours motion. Notably, 4D CT data (dynamic image volume) is a great improvement but seems currently to be only at a research stage in few institutes. To be used in treatment planning: 1- Tumours should be segmented semi-automatically on each image. Therefore it cannot be used conveniently. 2- Data are not provided during beam delivery. Consequently predicting lungs behaviour is quite speculative. Thus, further treatments are necessary.

The aim of our approach is to provide a parametric deformable model. The input of this model includes compliance, air flow, thoracic movements of each patient. The goal is to give a better prediction of lung motion to obtain a more precise dosimetric calculation. We chose to model lung by a 3D dynamic deformable model of continuous mechanics, computed with finite element method (FEM). This approach will allow a better lung mechanics behaviour understanding. Besides, different shape, position and size tumours could be virtually modelled and safety margins could be simulated, offering a physician learning.

In this paper, after a survey of lung parameters describing mechanical behaviour, we explain our approach and the numerical resolution insisting on nonlinearity problem; we conclude this part with a "technical" validation of our method. The numerical experiments section will concern the analysis of the mechanical parameters influence.

\section{Mechanical parameters for lung modelling survey}

Elastic properties of lung tissues are determined by their stress-bearing constituents: collagen and elastin fibres. Mead [8] showed that the static elastic properties of lung tissues are derived not only from the properties of the underlying tissue constituents but also from the way these constituents are organised. He showed that elastin is responsible for load bearing at low strains. As strain increases, the collagen fibres become straight and so progressively bear more load, stiffening the tissue. Fung et al. [12] assumed a linear expression for the constitutive equation for elastin together with an exponential function for collagen. This gave a tissue strain energy function from which the material constants were calculated with biaxial loading tests. This law is often included into finite element models like in the Yang and Wang modelling of human thorax [15], but the constant values can not easily be measured.

In various works, lung material is assumed to be elastic. Its mechanical properties are described with the Young modulus and the Poisson's ratio. A clinical test called compliance provides the ratio of air volume variation to the related air pressure variation. If alveolar surface tension is ignored, a link between this static compliance and the elastic properties could be established [3]. This pressure-volume curve provides then sufficient information to estimate bulk modulus. Poisson's ratio $(\nu)$ is quite difficult to measure. Using the technique of finite elements, West and Matthews [14] studied how lung is de- 
formed by its own weight. However, the lack of data on human lung tissues brought them to measure dog lung mechanical parameters. They found and used a mean value $\nu=0.3$. Lambert and Wilson [6] developed a mathematical model of elastic properties for a lung parenchyma considered as randomly oriented. Values of elastic moduli are obtained from the pressure-volume diagram for the whole lung. They obtained $\nu=0.25$. Lai-Fook [5] used two tests of uniaxial compression on dog lung lobes between parallel plates and punch indentations. They obtained $\nu=0.47$.

Although these data are very few and only belong to dogs, recent works refer to these three values. Alder et al. [1] used $\nu=0.49$ in a model taking into account mechanical and electrical properties. Owenet al. [10] used $\nu=0.3$ for a study of high-frequency ventilation influence on lung tissue. Grimal [4] used $\nu=0.3$ in his model of injuries modelling of thoracic impact.

Finally, various values of Poisson's ratio were measured or computed. The values vary from 0.25 to 0.47. In the section 5 we propose an estimation of the influence of these parameters through numerical simulations using our Model.

\section{Our approach}

Thorax (Fig 1) is the higher part of the trunk, which extends from the base of the neck to the diaphragm. The lower part of the trunk, the abdomen, is not considered. Twelve pairs of coasts of the rib cage protect thoracic organs. The thorax volume is mainly occupied by the pulmonary parenchyma - i.e lungs -, the heart, the bronchial tubes and the large vessels. Lungs are wrapped in a fibrous membrane: the pleura, containing a lubricating fluid which allows slipping. The pressure in the cavity is below the atmospheric pressure, making tissue to remain in contact. Diaphragmatic and rib-cage-muscle actions cause pressure changes inside the pleura, and consequently steadily induce lungs inflating or deflating. According to their location, lung tumours can undergo large displacements (heart beating, diaphragm and thorax displacement) [7].

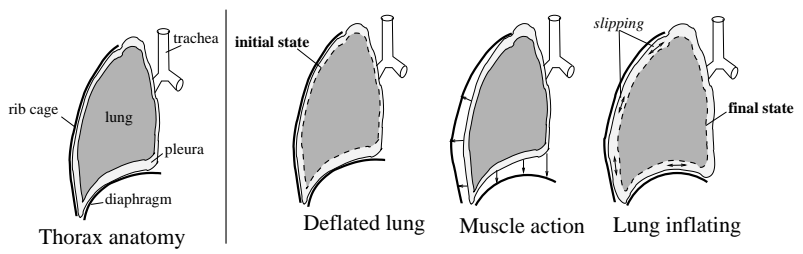

Figure 1. Lung sketch

To model lung inflation, we base our simulation on continuous mechanics formalism. Indeed, knowing the displacements at each point of the lung surface (corresponding to two different inflating states (cf Fig.1) it is possible to calculate the displacements of each point inside the lung. We plan to extract the surface displacements from thoracic external motion and from a diaphragm model monitored by some respiratory-cycle parameters (airflow, abdomen external motion). However, it does not give the individual motion of each point of the surface as required by continuous mechanics, but a global change of the lung surface. Consequently, there is no possible mapping.

Our strategy was then to simulate as precisely as possible the pleura behaviour (Fig.1): first, the whole system is fixed to the trachea. Then, we apply a uniform negative pressure around the lung at its initial surface, checking when the external surface matches the external surface of the lung at the next lung state of interest: it is realised by contact condition allowing sliding without friction as pleura does. It means that we do not search a point to point matching but a surface to surface matching between two states.

\section{Resolution}

Here, after a brief presentation of the lung geometry extraction, we present non-linearity problems we have to face during the simulation using FEM. The last part will concern results validating our approach.

\subsection{Lung geometry}

An experimental protocol has been defined by oncologists to provide us with thoracic CT scan examinations. During a CT-scan, organs can move and introduce artifacts. To reduce these uncertainties, we record the CT scan examination by determining a position of the respiratory cycle and by blocking the breathing in this position (ABC).

To extract lung surface, we segment CT scan sections of the right and left lungs and of the surrounding organs (Fig. 2).

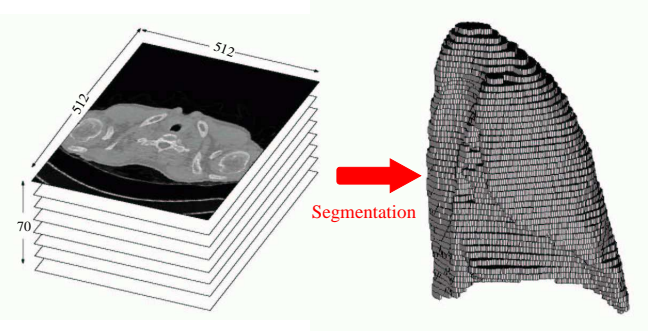

Figure 2. CT scan to Segmented lung

\subsection{Non-linearity problems}

Our physics based model is driven by continuous mechanics laws and then characterised by mechanical parameters. Continuum mechanics is a formalism adapted to predict the response of a solid material to applied stress. In a global approach, and as a first 
approximation, a homogeneous model is used to parameterise lung inflation. In the future, heterogeneity and other mechanical properties of true materials will be taken into account.

The FEM [16] is a numerical method that consists in approaching the solution by a simple expression based on the discretisation of the space into small elements. In our case it consists in searching displacements $U$ to reduce as possible the residue $R$ defined by (1).

$$
R(U)=F-K(U) \cdot U=0
$$

where $K$ is the stiffness matrix and $F$ is the load vector.

Practically the set of small elements is produced by a meshing process. Due to matter continuity, the convergence rate directly depends on mesh accuracy. We have presented in [13], a convenient method to generate a lung mesh. Currently, no computing time optimisation is searched.

\subsubsection{Large strains problem}

Commonly, lung volume can increase by a factor of two during a typical respiration cycle. The displacements are too large to assume that geometry changes will not influence the mechanical behaviour. Large strains have then to be considered. Therefore, we employ the iterative algorithm presented in [11] to take them into account. This method uses the Cauchy-Green strain tensor $\epsilon_{c g}$ computed with the transformation gradient $G$ of the geometrical deformation from a state $i$ to a state $f$ :

$$
\epsilon_{c g}=1 / 2\left(I d-\left(G \cdot G^{T}\right)^{-1}\right)
$$

with $I d$ the identity matrix, $G^{T}$ is the transposed matrix of $G$, which defines the metric change in the vicinity of a point. It is given by:

$$
G=I d+\operatorname{grad}_{X_{i}}(U)
$$

where $X_{i}$ represents the points positions within the studied solid at a state $i$ and $U$ is the displacement $\left(X_{f}-X_{i}\right)$.

The stress tensor used is the Kirchoff tensor $\tau\left(X_{f}\right)$, computed like a "scaling" of the tensor $\sigma\left(X_{i}\right)$ at the state $i$ :

$$
\tau\left(X_{f}\right)=\operatorname{det}(G) \cdot \sigma\left(X_{i}\right)
$$

where $\sigma\left(X_{i}\right)$ is the state of stress at the position $X_{i}$.

In our FEM approach, the non-linearity due to large displacements is solved with the NewtonRaphson algorithm. It is an iterative method defining a series of $\left(U_{n}\right)$ converging to the solution $U$ of equation 1. A Taylor series development of $R(U)$ in $\mathrm{U}$ at the vicinity of $U_{n-1}$ gives:

$$
R(U)=R\left(U_{n-1}\right)+\left(U-U_{n-1}\right) \frac{\partial R}{\partial U}\left(U_{n-1}\right)+O\left(\left(U-U_{n-1}\right)^{2}\right)
$$

with $\frac{\partial R}{\partial U}=\frac{\partial(K \cdot U-F)}{\partial U}=K$

If we set $U_{n}$ to be $R\left(U_{n-1}\right)+(U-$ $\left.U_{n-1}\right) K\left(U_{n-1}\right)=0$, then $R(U)=O\left(\left(U-U_{n-1}\right)^{2}\right)$. Finally, more iterations decrease $O\left(\left(U-U_{n-1}\right)^{2}\right)$ and the residue. $\left(U_{n}\right)$ has to be defined by the recurrent relation:

$$
K\left(U_{n-1}\right) \cdot \Delta U_{n}-R\left(U_{n-1}\right)=0
$$

with:

$$
\Delta U_{n}=U_{n}-U_{n-1}
$$

$K\left(U_{n-1}\right)$ is evaluated with (2) and (4), then the residue $R\left(u_{n-1}\right)$ is estimated according to $(1)$, which gives $U_{n}$ from (6).

Finally, during each Newton-Raphson iteration, the global stiffness matrix $K$ is computed with current $U_{n-1}$ value. Therefore, during each step various values will be evaluated: the transformation gradient, its determinant and the Cauchy-Green strain to take into account large displacements.

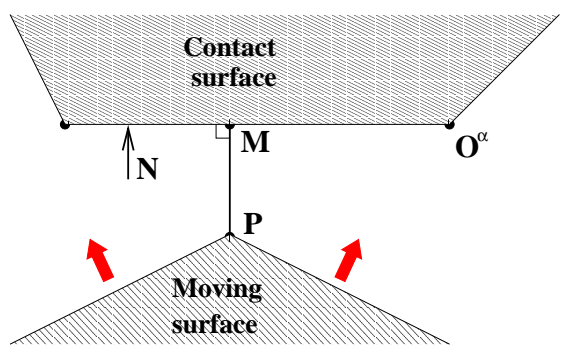

\section{Figure 3. Moving node projection on con- tact surface}

\subsubsection{Contact problem}

To handle contact conditions, couples of points $P$ and $M$ potentially in contact are searched: for each node $P$ of the moving surface, we define $M$, the projection of $P$ on the contact surface according to the direction of the surface normal vector $N$ (see Fig.3). Distance $P M$ must be positive to satisfy the conditions of non penetration, then dot product $P M . N \leq 0$ gives:

$$
P M_{n-1} \cdot N+\left(U_{M}-U_{P}\right) \cdot N \leq 0
$$

In eq.(8), $U_{M} \cdot N=0$ because $M$ is always on the contact surface. Then eq.(8) is equivalent to $U_{P} \cdot N \leq$ $P M_{n-1}$, which could be expressed as the following matrix form:

$$
\text { A.U } \leq d^{-}
$$

$A^{1}$ is called the matrix of contact and is built with the normal vectors. $d^{-}$is the distance $P M$ at the $n-1$ step. In Fig.3, we simply have $A=[N x N y]$.

\footnotetext{
${ }^{1}$ Practically, in our software matrix A takes into account general cases where both surfaces can independently move.
} 
Finally, the system to solve is composed of:

1) The non-penetration relationship: eq.(9).

2) The equilibrium equation completed by a force, eventually null, to add compression and avoid a separation. This force is $\mu . N$ for each couple in contact and $\mu$ is a Lagrangian multiplier. In the case of linear elasticity, the equilibrium equation becomes: $K . U+A^{T} \mu=F$ where $A^{T}$ is the transposed matrix of $A$.

3) The equation to express that this force only takes part when contact is reached and only corresponds to a compression force is: $\mu \geq=0$.

4) The last equation ensures compatibility : when there is contact $\mu$ is not null, and otherwise it is null: $\forall j,\left(A . U-d^{-}\right) \cdot \mu_{j}=0$.

If the imposed negative pressure is not sufficiently important, residue $R$ of equation (1) will be reached before contact condition. The pressure value must then be large enough.

The Newton-Raphson iterations are stopped when a fixed residue is reached. Nevertheless applied to a problem with contact condition the results are not satisfactory. Convergence is then ensured by subiterations taking into account geometry reactualisations. In other words, a subdivision is introduced and supported by the second index named $m$. $U_{n}$ is replaced by $U_{n, j}$. $n$ represents the current NewtonRaphson iteration and $j \in[1, m]$ represents succession of $m$ geometry reactualisation numbers for contact resolution. After each Newton-Raphson iteration, displacement variation $\Delta U$ is predicted. Contact conditions are then treated with various geometry reactualisations to obtain a new $\Delta U$ and update $U_{n+1}=U_{n}+\Delta U$.

The importance of the number $m$ of geometry reactualisations when contact occurs is then crucial for the accuracy of the final result. The more often the stiffness matrix is computed, the more exact the solution will be.

\subsection{Technical validation}

Our numerical simulations were carried out with the code-aster [2] finite element software. The tests have been realised on a Pentium 4, 2.40GH with $1 \mathrm{~GB}$ memory.

Experiments have been made with contact conditions as described in $\S 3$ to simulate real inflating.

A visual validation has been done to check if the final surface matches well with geometry extracted from CT scans. Fig. 4 shows that the maximum displacements occur where the final surface is far away from the initial surface. The final surface is the wireframe representation and the displacements are represented by a grey scale image set at the initial position.

\section{$5 \quad$ Numerical experiments}

Let us now focus on another kind of parameters in the simulation : the mechanical parameters. The im-

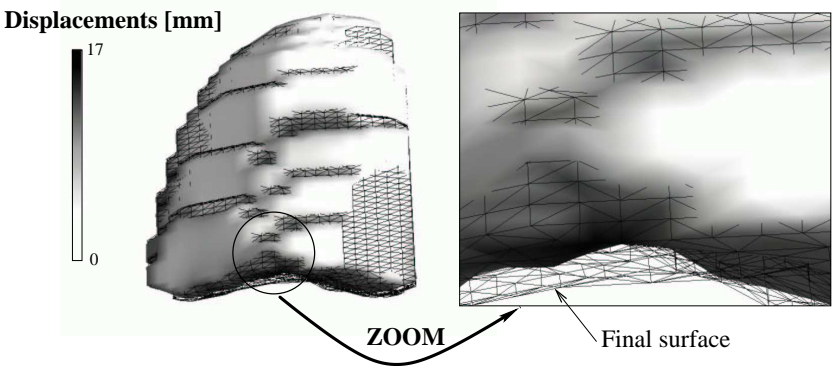

Figure 4. Detail of inflating with contact

portance of lung behaviour on such parameters have to be studied to evaluate the level of accuracy that is needed for a correct personalisation. During the evaluation we noted a relation between the computing time and the mechanical parameters values.

\subsection{Trial definition}

For such experiments, we fixed the same trial conditions except otherwhile stated. The mechanical parameters are the following : first geometry is always the same, extracted from the same patient, characterised by 10778 points and 30097 elements. Lung bounding box dimensions are $240 \mathrm{~mm} \times 180 \mathrm{~mm} \times$ $245 \mathrm{~mm}$. Patient compliance $C$ has been here measured to be $C=3.5 \mathrm{l} / \mathrm{kPa}$, initial volume of the studied lung is $V_{i}=3.6 \mathrm{l}$, its final volume is $V_{f}=3.8 \mathrm{l}$. According to compliance recoil law, pressure needed for a correct inflating of $V_{f}-V_{i}$ is given by:

$$
d P=\frac{-d V}{C}=\frac{3.8-3.6}{3.5}=57 \mathrm{~Pa}
$$

To apply a sufficiently large negative pressure as seen in $\S 4.2$, we set $P=60 \mathrm{~Pa}$.

Patient compliance $C=3.5 \mathrm{l} / \mathrm{kPa}$ with initial volume $V_{i}=3.6 l$ and $\nu=0.3$ gives $E=2 \frac{V_{i}(1-2 \nu)}{C}=$ $823 \mathrm{~Pa}$. It is the same value used in all our previous works.

Convergence parameters were also fixed. Minimum residue of equation 6 is set to $R=10^{-6}$ and the maximum iteration number is set to 99 . Computation of lung motion have been calculated with several numbers of geometry reactualisation for the contact conditions. We calculated the average and the standard deviation. After a series of tests with 1 to 7 reactualisations. We observed that a correct convergence can be obtained after 5 iterations (see Table.1) while error is lower than $2 \%$.

To analyse the numerical experiments in our medical context, we assume that influence of a mechanical parameter is not preponderant if differences between trial results is inferior to $5 \%$.

\subsection{Trial results}

From the state of the art previously discussed in $\S 2$ we know that different Poisson's ratios are often 


\begin{tabular}{|c|c|c|}
\hline $\begin{array}{c}\text { Reactualisation } \\
\text { number }\end{array}$ & $\begin{array}{c}\text { Computing } \\
\text { time }\end{array}$ & $\begin{array}{c}\text { Average } \\
\text { displacement }[\mathrm{mm}]\end{array}$ \\
\hline 1 & $14 \mathrm{~h} 43 \mathrm{~m}$ & 5.66 \\
\hline 3 & $15 \mathrm{~h} 15 \mathrm{~m}$ & 5.23 \\
\hline 5 & $15 \mathrm{~h} 28 \mathrm{~m}$ & 5.1 \\
\hline 7 & $15 \mathrm{~h} 48 \mathrm{~m}$ & 5.1 \\
\hline
\end{tabular}

Table 1. Level of stabilisation versus iteration number

considered. We carried out experiments with the following values: $\nu=0.25, \nu=0.3$ and $\nu=0.35$. Results show that the computing time increases with Poisson's ratio. Increasing $\nu$ increases shear stresses. It may induce complex deformations and more iterations could be necessary. The influence of $\nu$ close to 0.5 on the convergence of the solution is quite normal since it corresponds to incompressible materials. In the same way, the complex geometry of lung could drive the simulation to different results when changing $\nu$. Displacement vectors have then be studied by dividing results into two kinds : bulk displacements and surface displacements. The bulk displacements are measured on nodes of the hexahedral mesh and the surface displacements are measured on nodes of the external mesh. Experiments details are summed up in Table.2 and in Table.3. Are presented in Table.2 (respectively Table.3) the average displacement norms over bulk (respectively surface) positions for three different Poisson's ratio. To complete this average quantity we performed histograms of differences in displacement norms with respect to a reference (arbitrary set to 0.3). It allows us to calculate average and standard deviation. In the same way we characterised the angle between each displacement obtained for two Poisson's ratio.

The obtained results show that the value of Poisson's ratio is important. Notably, differences between simulations are more important on surface mesh. The average norm differences between bulk and surface displacement are obvious whereas angle differences are more significant in terms of standard deviation.

\begin{tabular}{|c|c|c|c|}
\hline Poisson's ratio & 0.25 & 0.3 & 0.35 \\
\hline Computing time & $14 \mathrm{~h}$ & $16 \mathrm{~h}$ & $30 \mathrm{~h}$ \\
\hline $\begin{array}{c}\text { Average displacement } \\
\text { in [mm] }\end{array}$ & 5 & 4.5 & 3.9 \\
\hline $\begin{array}{c}\text { Average norm difference } \\
\text { with } \nu=0.3\end{array}$ & 0.5 & $/$ & 0.6 \\
\hline $\begin{array}{c}\text { Standard deviation } \\
\text { in norm with } \nu=0.3\end{array}$ & 0.35 & $/$ & 0.4 \\
\hline $\begin{array}{c}\text { Average angle difference } \\
\text { with } \nu=0.3\end{array}$ & $6^{\circ}$ & $/$ & $11^{\circ}$ \\
\hline $\begin{array}{c}\text { Standard deviation } \\
\text { in angle with } \nu=0.3\end{array}$ & $5^{\circ}$ & $/$ & $10^{\circ}$ \\
\hline
\end{tabular}

Table 2. Poisson's ratio experiment table with bulk displacements

\begin{tabular}{|c|c|c|c|}
\hline Poisson's ratio & 0.25 & 0.3 & 0.35 \\
\hline Computing time & $14 \mathrm{~h}$ & $16 \mathrm{~h}$ & $30 \mathrm{~h}$ \\
\hline $\begin{array}{c}\text { Average displacement } \\
\text { in [mm] }\end{array}$ & 5.8 & 5.1 & 4.4 \\
\hline \hline $\begin{array}{c}\text { Average norm difference } \\
\text { with } \nu=0.3\end{array}$ & 0.7 & $/$ & 0.7 \\
\hline $\begin{array}{c}\text { Standard deviation } \\
\text { in norm with } \nu=0.3\end{array}$ & 0.4 & $/$ & 0.5 \\
\hline $\begin{array}{c}\text { Average angle difference } \\
\text { with } \nu=0.3\end{array}$ & $6^{\circ}$ & $/$ & $9^{\circ}$ \\
\hline $\begin{array}{c}\text { Standard deviation } \\
\text { in angle with } \nu=0.3\end{array}$ & $9^{\circ}$ & $/$ & $15^{\circ}$ \\
\hline
\end{tabular}

\section{Table 3. Poisson's ratio experiment table with surface displacements}

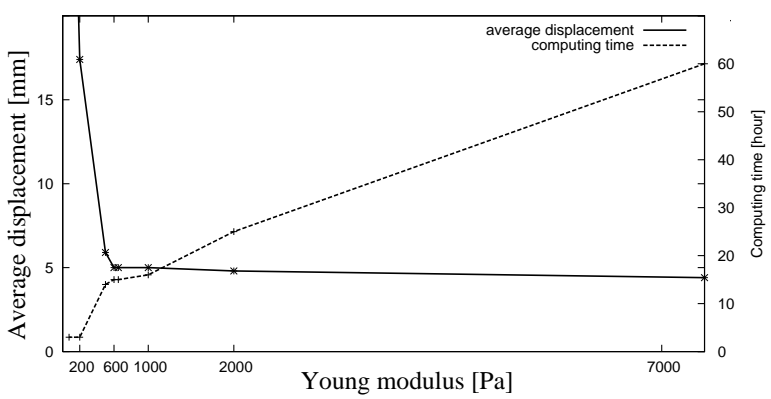

Figure 5. Comparison of Young modulus with differences in norm

We performed tests to study Young modulus influence on lung behaviour within in our assumption of heterogeneity of lung tissue. On the figure 5 the solid line represents different average displacements as a function of $E$. For low values, lung tissue is considered to be very soft. After a single load increment, contact surface is exceeded and contact will no longer be checked. Around our estimate value of Young modulus from compliance, the average displacement is almost constant. With large values of Young modulus, lung tissue is so hard that contact possibly cannot occur (because the negative pressure is not high enough). The regime of constant displacements is actually obvious. Indeed, the continuous equations are linear with regards to Young modulus. So, except for the convergence problems for small values of $E$ and the lack of negative pressure for high value of $E$, the displacements are only function of initial and final states. The dash line represents the computing time. For low value of $E$, there is no contact treatment therefore the computing time is very fast. Contact treatment strongly depends on the number of contact nodes. For high values of $E$, each newton iteration step implies very small displacements. The end of the simulation occurs after a great number of load steps; therefore after an important computing time.

Finally, in principle Young modulus can be arbitrary chosen provided it is lower than the value that 
ensures a contact situation. Practically, convergence troubles and computing time constraint imply optimal choice.

\section{Conclusion}

We propose a model of lung inflating based on continuous mechanics and considering global properties for the lung. When solving the equation with finite element method we observe the crucial importance of meshing. In this paper, we fulfilled the validation of our meshing methodology and performed tests of convergence that ensure the stability of our code.

Besides, we evaluated the influence of the mechanical parameters of our model namely Poisson's ratio and Young modulus. We showed that in principle Young modulus can be chosen arbitrary provided the negative pressure is high enough. Practically we observed that an optimal choice of its value with regard to the negative pressure avoids convergence problem and reduces computing time. Contrary to other statements in some papers, we showed that the Poisson's ratio plays a significant role and may not be arbitrarily chosen for a correct prediction.

\section{$7 \quad$ Future works}

First of all, to use this work in clinical context the geometry has to be converted into CT scan images for treatment planning. To evaluate our model validity a study will estimate the difference between simulated CT scans obtained from our model and the data from real CT scans. Finally, if necessary, modelling will be performed in a more realistic way. A protocol is under elaboration to measure elasticity on various samples of a same lung tissue. Heterogeneity and non-linear elasticity law will be added through the lung mechanic properties.

\section{Acknowledgements}

We thank the ARC (the French Association for Cancer Research) for their financial support and all our partners: the Léon Bérard Centre and the ETOILE ${ }^{2}$ project (Espace de Traitement Oncologique par Ions Légers) for their support.

\section{References}

[1] A. Adler and R. Guardo. Electrical impedance tomography: Regularised imaging and contrast detection. IEEE Trans. Medical Imaging, 15:170-179, 1996.

[2] Code Aster. http://www.code-aster.org/.

[3] V. Baudet, P-F. Villard, F. Jaillet, M. Beuve, and B. Shariat. Towards accurate tumour tracking in lungs. IEEE, Mediviz, Conference on Information Visualization, pages 338-343, 2003.

\footnotetext{
${ }^{2}$ http://ETOILE.univ-lyon 1.fr
}

[4] Q. Grimal, A. Watzky, and S. Naili. Nonpenetrating impact on the thorax : a study of the wave propagation. Comptes Rendus de l'Académie des Sciences, IIb(329):655-662, 2001.

[5] SJ Lai-Fook. Lung parenchyma described as a prestressed compressible material. J Biomech., 10(5-6):357-65, 1977.

[6] RK. Lambert and TA. Wilson. A model for the elastic properties of the lung and their effect of expiratory flow. J Appl Physiol., 34:34-48, 1973.

[7] K. M. Langen and D. T. L. Jones. Organ motion and its management. Int. J. Radiation Oncology Biol. Phys., 50(1):265-278, 2001.

[8] J Mead. Mechanical properties of lungs. Physiol. Rev., 41:281-328, 1961.

[9] Marylin L. Moy and Stephen H. Loring. Compliance. Seminar in respiratory and critical care medecine, 19(4):349-359, 1998.

[10] M.R. Owen and M.A. Lewis. The mechanics of lung tissue under high-frequency ventilation. SIAM Journal on Applied Mathematics, 61(5):1731-1761, 2001.

[11] J.C. Simo and C. Miehe. Associative coupled thermoplasticity at finite strains: formulation, numerical analysis and implementation. Comp. Meth. Appl. Mech. Eng., 98:41-104, 1992.

[12] D.L. Vawter, Y.C. Fung, and J.B. West. Constituve equation of lung elasticity. Journal of Biomechanical Engineering, (101):38-45, 1979.

[13] P-F. Villard, M. Beuve, B. Shariat, V. Baudet, and F. Jaillet. Lung mesh generation to simulate breathing motion with a finite element method. IEEE, Mediviz, Conference on Information $\mathrm{Vi}$ sualization, pages 194-199, 2004.

[14] J.B. West and F.L. Matthews. Stresses, strains, and surface pressures in the lung caused by its weight. J. Appl. Physiol., 32:332-345, 1972.

[15] K.H. Yang and K.H. Wang. Finite element modeling of the human thorax. The Second Visible Human Project Conference, 1998.

[16] O. C. Zienkiewicz. La Méthode des éléments finis. McGraw Hill Montréal Paris, 1979. Traduction de la 3ème édition anglaise. 